\title{
Identification of Superior Allele for Drought Responsive $M y b$ Gene in different Germplasms of Eleusine coracana through Morpho-Physiological, Biochemical and Sequence Diversity Analysis
}

\author{
Akash Sinha, Megha Bhatt, Ankita Chauhan and Pushpa Lohani*
}

Department of Molecular Biology and Genetic Engineering, College of Basic Science and Humanities, G. B. Pant University of Agriculture and Technology, Pantnagar, India

*Corresponding author

\section{A B S T R A C T}

\section{Keywords}

Allele, Drought tolerance, morhophysiological, biochemical, sequence diversity, myb gene, SNP

Article Info

\section{Accepted:}

12 March 2021

Available Online:

10 April 2021
Abiotic stresses like drought, salinity and cold cause huge losses to crop yield. There is an urgent need to produce drought tolerance in crops in order to meet challenges posed by changing climatic conditions and food security. One of the way in which the challenge could be met is by fishing out superior allele of a drought responsive gene and its utilization in producing drought tolerance in crops. The present study was carried out with the objective to study the variation in morpho-physiological and biochemical parameters of 11 day drought stress treatment on different genotypes of finger millet at the vegetative stage and determine the most drought tolerant genotype. Myb gene sequence of the tolerant genotype was considered as superior allele for drought tolerance. Therefore, DNA was isolated from 12 genotypes of E. coracana and PCR amplification was performed using gene specific primers. The amplicons were sequenced and subjected to further analysis using various bioinformatics tools. Multiple Sequence Analysis results indicated that considerable nucleotide diversity was present in the drought responsive Myb gene sequence in the finger millet germplasm. PRM-6107 was found to be the most drought tolerant genotype, amongst all the genotypes under study based on the various morpho-physiological and biochemical tests. PCPGR-8115 was observed as the most sensitive genotype. Myb gene sequence present in PRM-6107 was considered as superior myb allele. Single nucleotide polymorphisms and insertions-deletions were observed in the myb allele of PRM-6107.

\section{Introduction}

Drought is a major abiotic stress that culminates in severe loss of yield to crops and has proven to be more scourging than any other physical hazard.
In order to overcome such problem an important approach is to generate crops with enhanced drought tolerance, by utilizing biotechnology and molecular biology techniques. This strategy requires the 
identification of traits relating to drought tolerance and then of the genes that determine these traits. Plants often carry genes that enable them to maintain their performance despite of severe moisture stress conditions. The genetic and molecular basis of drought tolerance has been investigated extensively and genes encoding drought-related transcription factors and functional proteins have been identified. These stress-inducible genes can be classified into two groups. The first group includes proteins that most probably function in abiotic stress tolerance. These include molecules such as chaperones, late embryogenesis abundant (LEA) proteins, osmotin, antifreeze proteins, mRNA-binding proteins, key enzymes for osmolyte biosynthesis, water channel proteins, sugar and proline transporters, detoxification enzymes, and various proteases. The second group is comprised of regulatory proteins. These include various transcription factors, protein kinases, protein phosphatases, enzymes involved in phospholipid metabolism, and other signaling molecules such as calmodulin-binding protein. Regulatory RNAs including siRNAs and miRNAs have also been discovered as important regulators in drought stress response and tolerance.

This leads to the observation that drought tolerance is a quantitative trait involving complex genetic control as it is multigenic. Therefore research on the master regulators that modulate the expression of all such genes gained limelight. These master regulators are the transcription factors that are induced on the onset of drought conditions and bind specifically to the promoters of such tolerance related genes. An important transcription factor amongst these is the MYB transcription factor. The MYB proteins are characterized by a MYB DNA binding domain in their $\mathrm{N}$ terminus that is composed of one or more imperfect tandem repeat(s) (Riechmann et al.,
2000). MYB with the R2R3 type repeat is found in plants and have been reported to be responsible for regulating the expression of ABA mediated drought tolerance related genes (Jung et al., 2008). They have been found to have specific role in response to water stress, such as the regulation of stomatal movement, the control of suberin and cuticular waxes synthesis and the regulation of flower development. A member of the MYB family has been identified in finger millet and has been reported to be up-regulated on treatment of drought (Salvi et al., 2012). India is the secondary centre of origin for finger millet. Thus, a number of genotypes of finger millets can be found in India, of which only a fraction is cultivated today. Such uncultivated varieties, wild relatives and landraces are believed to possess alleles that are more potent in combating drought stress. A number of alleles of the identified $M y b$ genes ought to be present in different genotypes of finger millet. Intensive breeding efforts have concentrated the favorable alleles already selected during early domestication and thus contributed to further narrowing of the gene pool (Ladizinsky 1985; Simmonds 1976). Wild relatives of cultivated plants didn't have to suffer from bottle necks or selective sweeps. But the cultivated plants had to undergo these processes during the course of domestication, when suitable traits were selected for improvement. Thus it can be safely presumed that the wild gene pools, like landraces, are intact and conserve much of the variation present originally. This is supported by the fact that wild relatives are often better adapted to stressful conditions than their cultivated versions (Cortés et al., 2012). It is important to use this variation for the identification and isolation of novel and superior alleles of such agronomically important genes from crop gene pools to suitably deploy for the development of improved cultivars. Germplasm collections worldwide have immense unexploited allelic variations of such 
genes. Consequently it can be expected that the traits that were not subjected to diversifying selection or genes that are part of the domestication syndrome, the wild relatives have higher genetic diversity as compared to cultivated ones. This trend has been demonstrated in studies on crops like rice ( $\mathrm{Li}$ et al., 2011). Purifying selection and local adaptation are what most commonly observed in analysis of wild and cultivated varieties. Mutations in coding regions have an excellent effect on the phenotype through changing the particular encoded protein structure as well as function. Progress in breeding for superior high yielding varieties of agricultural crops was made possible by accumulation of beneficial alleles from varying plant genetic resources existing worldwide. Still, a large proportion of the beneficial/superior alleles have not been utilized as these were left behind in the wild ancestors of cultivated species during domestication. The untapped genetic variation existing in the wild relatives and land races of crop plants could be exploited gainfully for development of agronomically superior cultivars.

Deciphering such untapped useful nucleotide diversity patterns for drought-related genes can be performed by nucleotide diversity study. Nucleotide diversity study is a useful strategy for rapid characterization of diversity stored in gene bank accessions at a genetic locus of agronomical importance (Bhullar et al., 2010). Eco-TILLING and sequence based nucleotide diversity study are two approaches followed to identify nucleotide polymorphisms for a candidate gene in populations retrieved from gene banks. Such allele discovery helps in use of such genotypes in conventional breeding programs, development of allele specific markers, new haplotype identification and in tracing the story of allele evolution and the relationships between them. The recent advancements made in the field of next generation sequencing have made the approach of nucleotide diversity study less cumbersome, more practicable and cheaper.

This study aims to study the nucleotide diversity and fish out the superior alleles of an already established drought tolerance gene, Myb, present in the germplasm of finger millet.

\section{Materials and Methods}

\section{Plant material}

Seedlings of different accessions of finger millets were used for experiments. The accessions were PCPGR-8064, PCPGR-8065, PCPGR-8067, PCPGR-8110, PCPGR-8112, PCPGR-8113, PCPGR-8114, PCPGR-8115, PCPGR-8116, PCPGR-8123, PES-400 and PRM-6107. The seeds were obtained from the Pantnagar Centre for Plant Genetic Resources (PCPGR), Pantnagar which were collected by the centre from different regions of Uttarakhand.

All the accessions were uncharacterized. Seeds of all the genotypes of finger millet were sown in trays filled with soil and vermicompost mixture in the ratio $2: 1$. Small plants of about $10 \mathrm{~cm}$ height were transferred to pots and kept in a polyhouse. They were allowed to grow in controlled polyhouse conditions at optimum temperature and moisture for 45 days.

\section{Drought stress imposition and phenotyping for drought tolerance}

After the plants reached their seedling stage, drought stress was imposed on the plants of treatment pots by stopping irrigation for 11 days, while the control pots were regularly irrigated to keep the soil moisture content at $60 \%$ of the total water retaining capacity. The plants were phenotyped and compared with 
the control for the following physiological and biochemical parameters following the 11 day drought treatment.

\section{Morpho-Physiological parameters}

Shoot Length, Root length, Number of Tillers, Number of leaves, Leaf Rolling and Drying, Leaf area, Relative water content, chlorophyll content and total carotenoid content. Leaf area of leaves from both control and treatment plants of all genotypes was measured by the method described by Rajappa et al., (1972). A factor of 0.75 multiplied by the product of the length and width of the flag leaf was used to determine the leaf area of both control and drought treated plants expressed in $\mathrm{cm}^{2}$. Relative water content (RWC), considered the appropriate measure of plant water status in terms of the physiological consequence of cellular water deficit was estimated by the method of Barrs and Weatherley(1962) from the topmost healthy and unblemished leaves collected at 11 AM. The assay for chlorophyll content was performed based on the various equations for the determination of pigments based on their absorption in organic solvents at various wavelengths as described by Lichtenthaler and Wellburn(1983). $50 \mathrm{mg}$ leaf material was placed in $10 \mathrm{ml}$ of Dimethyl sulfoxide (DMSO) in test tubes, and thereafter the test tubes were placed in a constant temperature incubator at $65^{\circ} \mathrm{C}$ for 4 hours. After the completion of period the tubes were cooled to room temperature. The absorbance of both the solvents was recorded at 470, 645 and $663 \mathrm{~nm}$ against DMSO blank.

\section{Biochemical parameters}

Proline content was estimated using the acid ninhydrin method devised by Bates et al.,(1973). Malondialdehyde content was also estimated. Lipid peroxidation is oxidative degradation of lipid-fatty acids by reactive oxygen species. The level of lipid peroxidation was measured in terms of thiobarbituric acid reactive substances (TBARS) content, which includes products like malondialdehyde, fatty acid-hydroperoxides, by the method given by Heath and Packer (1968).

Gravimetric method of soil moisture content determination was used

\section{DNA isolation}

Genomic DNA isolation from the leaves from all the genotypes was performed according to the method described by Murray and Thompson(1980)using the Extraction buffer having the following components: $100 \mathrm{mM}$ Tris-Cl (pH 8.0), 20mM EDTA (pH 8.0), 1.4 $\mathrm{M} \mathrm{NaCl}, 2 \%$ (w/v) CTAB (Cetyltrimethyl ammonium bromide). RNase A treatment (50 $\mu \mathrm{g} / \mathrm{ml}$ ) was done to the DNA sample. The quality of DNA was checked by agarose gel electrophoresis on $1.2 \%$ gel in $1 \mathrm{X}$ TAE buffer and quantification was done by spectrophotometric method at $260 \mathrm{~nm}$.

\section{Primer Designing}

Gene specific primers were designed using full length sequence of Myb gene which was obtained by performing 5, RACE and 3'RACE on the partial CDS of Myb gene that was sequenced (MYB-JN107890)in previous studies (Salvi et al., 2012). RACE fragments were sequenced and aligned. Largest open reading frame was used for primer designing. Primers were designed using the online tool Primer3 and custom synthesized from Sigma company.

\section{DNA amplification}

The PCR reactions were performed in a $25 \mu \mathrm{l}$ final volume mixture containing $100 \mathrm{ng}$ of purified genomic DNA, 1X PCR buffer, 2.5 $\mathrm{mM}$ dNTPs, $0.4 \mu \mathrm{M}$ each of forward and 
reverse primers having sequence CCTTGCTTTCCACTGAAATCTCC and AACATACTTTTCTATGCATTCTGCTGCA CT respectively, $2.5 \mathrm{mM} \mathrm{MgCl} 2$, and $1.25 \mathrm{U}$ of Hi-proof DNA polymerase with 3'-5' exonuclease activity provided by HiMedia Laboratories for each reaction.

Initial Denaturation was done at $95^{\circ} \mathrm{C}$ for 5 min. The polymerization steps, Denaturation at $95^{\circ} \mathrm{C}$ for $45 \mathrm{sec}$, Annealing at $57^{\circ} \mathrm{C}$ for 45 sec and Extension at $72^{\circ} \mathrm{C}$ for 2 min were repeated for 35 cycles. Final extension was performed at $72^{\circ} \mathrm{C}$ for $10 \mathrm{~min}$. After the completion of the PCR cycles, the PCR product was checked for amplification by running on an electrophoresis assembly with $1.2 \%$ agarose gel at 70 volts and documented in a gel documentation system. The desired amplicons were eluted from the agarose gel by using Invitrogen gel extraction kit.

The eluted product was analyzed on a gel and after confirmation sent for sequencing to National Research Centre on Plant Biotechnology, New Delhi. Next Generation Sequencing was performed by Oligonucleotide Ligation and Detection technique (SOLiD), using 3730xl DNA Analyzer of Applied Biosystems.

\section{In silico Analysis}

The sequences, obtained after sequencing, were analyzed by multiple sequence alignment using MEGA version 7 (Kumar et al., 2016).

\section{Results and Discussion}

The effect of 11 day drought stress treatment on various genotypes of finger millet was studied at the vegetative stage. All the genotypes were uncharacterized and were collected by PCPGR, Pantnagar from different regions of Uttarakhand. In order to screen the different genotypes for tolerance to drought they were subjected to water deficit stress at 45 days after sowing by withholding irrigation for a period of 11 days. Soil moisture content of the treatment pots was determined and was observed to be around $20-25 \%$ in all the drought imposed pots. Drought stress was imposed on 45 day old plants and based on various morphological, physiological and biochemical parameters they were evaluated for drought tolerance. The control plants were evaluated in parallel. In this study it was observed that all the genotypes responded differently to different parameters of drought stress tolerance. Results were obtained based on mean values of 3 replicates of the experiments. For all the parameters the significant difference amongst the genotypes was found to be $\mathrm{P}<=0.05$.

\section{Morpho-physiological parameters studied to evaluate the effect of drought stress}

Plants which appeared similar in terms of morphological features were separated into 2 groups: Control and treatment, and drought stress was imposed on the treatment group.

After the 11 day drought period the treatment plants were analyzed and PRM 6107 was found to have the highest shoot length, number of tillers, leaf number, leaf rolling and drying, relative water content, total chlorophyll content and total carotenoid content. Root length and root to shoot ratio was found to be greater for PCPGR 8123, but was closely followed by PRM 6107. PCPGR 8115 showed the lowest values for most of the above parameters. PES 400 also had lower values. A higher value of these indices is a sign of higher tolerance to drought stress.

\section{Shoot Length}

Drought stress impacts severely the morphology of a grass plant. Cell enlargement is more drastically affected compared to cell 
division. The most important effects are exhibited in the form of decreased growth rate and reduced leaf area (Pande and Singh 1985). Kramer (1969) described this as a typical response of crop plants when exposed to drought conditions.

The shoot length was measured from soil layer up to the base of the flag leaf. Height of all the plants of the 12 genotypes was found to be lowered when compared with the control plants. However the shoot length of PRM6107 treatment plants was found to be highest as compared to the plants of other genotypes (Fig. 1). Inamullah et al., (1999), reported about significant reduction in plant height under moisture stress when compared to control conditions. The decrease in height under water deficit conditions is often either due to inhibition of cell division or cell expansion. The genotypes that are least affected by such inhibitions can be categorized as drought tolerant.

\section{Root Length}

The root system functions not only by supporting the above ground parts of a plant, but also by accessing water sources far below into the soil profile and obtaining water for the plant. The first organs sense water shortage are the roots. Its morphological and physiological properties largely determine the ability and rate of water and nutrient uptake. Traditionally root length density and depth are regarded as an important criteria for determining tolerance of plant root system (Kashiwagi et al., 2006). Li et al., (2011) reported that total root length is a major contributor to root architecture variation in different genotypes and can be used as an important selection criteria drought tolerance at the seedling stage. PCPGR-8123 had the highest root length amongst the drought treated plants, and it was closely followed by PRM-6107. In fact PCPGR-8123 and PRM-
6107 drought treated plants exhibited a higher root length than the control plants after the 11 day drought period (Fig. 2). This shows that the PCPGR-8123 and PRM-6107 possessed the capability to bring out drastic morphological alterations with regard to root system architecture in response to drought stress. Tomar et al., 2016, based on the study of drought tolerant cultivars of wheat reported that drought tolerant cultivars had a compact root system with higher depth as observed in the case of PCPGR-8123 and PRM-6107, while sensitive cultivars had lesser depth and higher horizontal root spread as was observed with PCPGR-8115 in this study.

\section{Root length to shoot length ratio}

The ratio of root to shoot length is used as an important parameter to evaluate drought tolerance in plants because deep rooted systems extract more water while relatively smaller shoots transpire less (Srividya et al., 2011). PCPGR-8123 was found to have the highest root to shoot length ratio, closely followed by PRM-6107. PCPGR-8116 had the least root to shoot length ratio (Fig. 3), which indicates PCPGR-8116 plants lacked the capability to reallocate its metabolites from the shoot to the root system, so that the roots could grow into deeper soil profiles in search of water. This is important for plants to survive in case of prolonged drought stress conditions (Rich and Watt 2013).

\section{Number of Tillers}

Bouman and Toung (2001) studied the responses of various genotypes during drought stress and reported that drought before or during the tillering stage leads to reduction in the number of tillers in a plant. Drought stress was found to have the most severe impact on the tillering of PES-400, while PRM-6107 was the least affected. Turner and Begg reported that decrease in soil moisture availability was 
directly related to decrease in the number of tillers(Turner and Begg 1978). Thus the plants which were able to maintain their tillering ability were considered to be more tolerant to drought than others. PCPGR-8116 showed tiller formation on the imposition of drought stress (Fig. 4). This may in confirmation with the findings of Fang et al., (2015), who reported that some plants often tend to escape stressful conditions by completing their life cycle as early as possible.

\section{Number of Leaves}

Leaves from three randomly selected plants for all the 12 genotypes in both the control and drought treated plants were counted. Leaf growth was most sensitive to water stress in PCPGR-8115 as its treatment plants were found to have the least number of leaves. Drought stress was found to have the least effect on the plants of PRM-6107 with regard to leaf growth because there was very little difference between the leaf number of control and treatment plants (Fig. 5). From the results it is evident that new leaf production is extremely sensitive to soil moisture availability since the control plants possessed higher number of leaves than the drought treated plants in all cases.

\section{Leaf Area}

Leaf area has direct relation with the photosynthetic capacity of a plant. Also drought stress severely affects the leaf area of plants(Potter and Jones 1977). Pande and Singh(1985)reported that cell expansion is severely affected in case of prolonged drought stress, which manifests in the form of reduced leaf area. Hence the plants that are able to maintain their leaf area despite drought conditions ought to be considered more drought tolerant than others. Compared to other genotypes, PRM-6107 and PCPGR-8123 exhibited higher leaf area. This indicates they are better able to maintain their leaf area and hence physiological processes like photosynthesis than others. PCPGR-8115 exhibited least leaf area than others and so can be considered sensitive according to this parameter (Fig. 6).

\section{Leaf rolling and drying}

Higher number of green leaf retention is an important characteristic feature of drought stress tolerant plant (Blum 2005). Leaves roll and dry as a result of moisture deficit conditions. Leaves roll to minimize the leaf surface area in order to reduce the amount of water lost by evapotranspiration. Rolling induced just at the onset of moisture stress is generally displayed by drought sensitive plants. Leaf rolling in such a case is induced as a result of poor osmotic adjustments and loss of turgor (Hsiao et al., 1984). Thus the area for photosynthesis gets severely reduced. Amongst the treatment plants least leaf rolling and drying was observed in PRM-6107, PCPGR-8067 and PCPGR-8123 indicating they are the most tolerant to drought stress with regard to leaf rolling and drying, while PCPGR-8115 is the most sensitive as it showed the highest rolling and drying in leaves when exposed to drought stress (Table $1)$.

\section{Relative Water Content}

Relative Water Content estimates the current water content of the sampled leaf tissue relative to the maximal water content it can hold at full turgidity. Larbi (2004) reported that relative water content can be used as an effective screening method to determine the level of drought tolerance in crops. The results indicate that drought stress resulted in the reduction in the relative water content in all the genotypes. However, PRM-6107 was able to maintain a higher RWC status as compared to other genotypes. This shows that there is a 
better maintenance of water status in PRM6107 , owing to osmotic adjustments as a result of drought stress. This indicates that PRM6107 has a better ability to counter drought conditions by bringing out osmoregulatory alterations in the plant tissue. PCPGR-8115 turned out to be the most sensitive genotype based on RWC, since it was not able to maintain its water status in response to drought stress (Fig. 7).

\section{Chlorophyll content}

Huge reduction in the chlorophyll content was observed in the leaves of all treatment plants of genotypes when compared to that of control plants. PRM-6107 was found to have the highest total chlorophyll content, followed by PCPGR-8123. PCPGR-8115 drought treated plants exhibited least chlorophyll content as compared to other genotypes (Fig. 8). Shmat'ko et al.,(1977) reported that drought tolerant genotypes show little change in the chlorophyll content compared to drought susceptible genotypes. Leaf chlorophyll decline as a consequence of water stress is a common observation(Chakraborty et al., 2001; Kar and Sinhababu 2002). Green leaf retention under drought conditions is considered to be an important parameter to select for drought tolerance(Datta et al., 1988). The results obtained convey that PRM-6107 is more capable to maintain its total chlorophyll content and hence perform photosynthetic processes in a more better way than others.

\section{Carotenoid content}

Carotenoids exhibit multifarious roles in drought conditions which involve light harvesting and oxidative damage protection. Farooq et al.,(2009) reported that beta carotene might be performing a major protective role in the photosynthetic tissue buy directly quenching the triplet chlorophyll and thus preventing the formation of singlet oxygen. Thus an increased carotenoid content in a genotype is an important indicator of stress tolerance. Total carotenoid content from randomly collected leaves of all genotypes was calculated. It was found drought stress caused reduction in the carotenoid content of all genotypes. However, PRM-6107 has the highest total carotenoid content amongst the treatment plants, followed by PCPGR-8067 (Fig. 9).

\section{Biochemical parameters studied to evaluate the effect of drought stress}

Proline content and malodialdehyde content was measured in the plants subjected to moisture stress treatment. PCPGR 8067 was recorded to have the highest proline content, and was followed by PRM 6107. In case of malondialdehyde content also, which is a product of lipid peroxidation, PRM 6107 fared the best by recording the lowest level of MDA in its leaves while PCPGR 8115 had the highest MDA content reflecting its drought susceptible nature.

\section{Proline content}

In this experiment it was observed that drought stress caused significant increase in the proline content, an important osmolyte.

The proline accumulation observed makes it evident that increased accumulation of proline is an adaptive response of plants to counter drought stress in confirmation with the findings of Sultan et al.,(2012). All the genotypes exhibited low free proline content in the control plants except PCPGR-8067 which contained a higher level of proline as compared to other genotypes. After the 11 day drought stress, all the genotypes showed a significant rise in their free proline content, with highest in PRM-6107 which thus is more capable to bring about osmotic adjustments to counter drought, followed by PCPGR-8123. PCPGR-8115 and PES-400 treatment plants exhibited the lowest proline content (Fig. 10). 
Table.1 Effect of drought stress on leaf rolling and drying

\begin{tabular}{|l|c|c|}
\hline Sl. & Genotypes & Rolled and Dried Leaves \\
\hline $\mathbf{1 .}$ & PCPGR-8064 & +++ \\
\hline $\mathbf{2 .}$ & PCPGR-8065 & + \\
\hline $\mathbf{3 .}$ & PCPGR-8067 & +++ \\
\hline $\mathbf{4 .}$ & PCPGR-8110 & ++ \\
\hline $\mathbf{5 .}$ & PCPGR-8112 & ++++ \\
\hline $\mathbf{6 .}$ & PCPGR-8113 & +++ \\
\hline $\mathbf{7 .}$ & PCPGR-8114 & +++ \\
\hline $\mathbf{8 .}$ & PCPGR-8115 & +++++ \\
\hline $\mathbf{9 .}$ & PCPGR-8116 & ++ \\
\hline $\mathbf{1 0 .}$ & PCPGR-8123 & + \\
\hline $\mathbf{1 1 .}$ & PES-400 & ++++ \\
\hline $\mathbf{1 2 .}$ & PRM-6107 & + \\
\hline
\end{tabular}

Legend

- $\quad+$, Upto 4 Leaves Rolled and Dried

- $\quad+$, Upto 8 Leaves Rolled and Dried

- $\quad+++$, Upto 12 Leaves Rolled and Dried

- $\quad++++$, Upto 16 Leaves Rolled and Dried

- $\quad+++++$, More than 16 Leaves Rolled and Dried

Fig.1 Effect of drought on shoot length

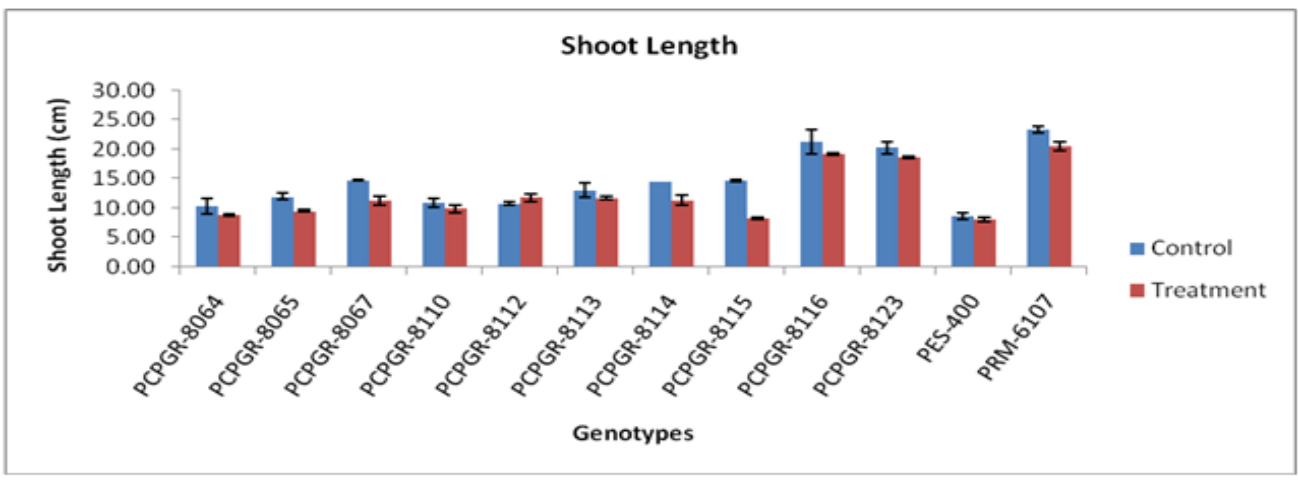

Fig.2 Effect of drought on root length

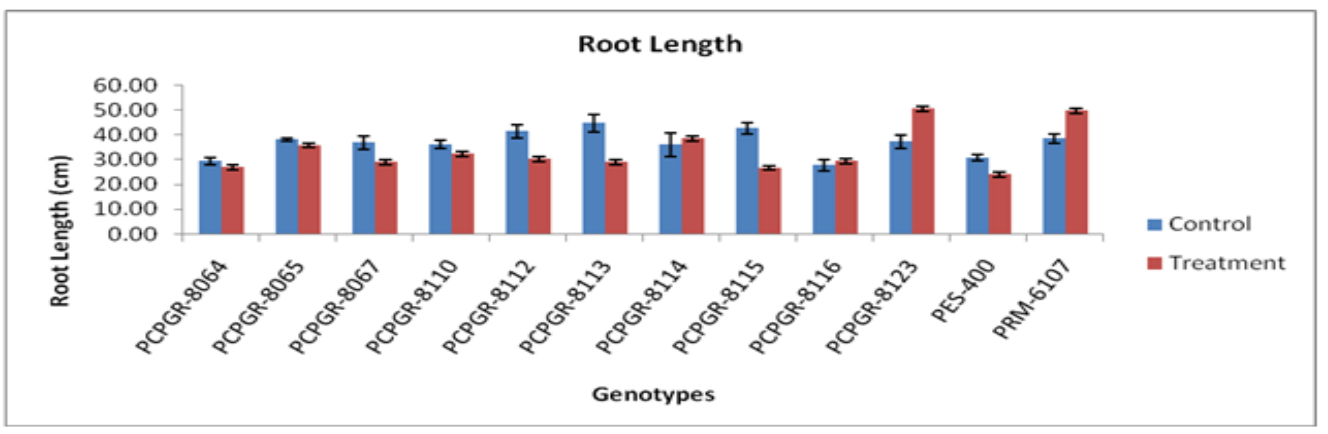


Fig.3 Effect of drought stress on root to shoot length ratio

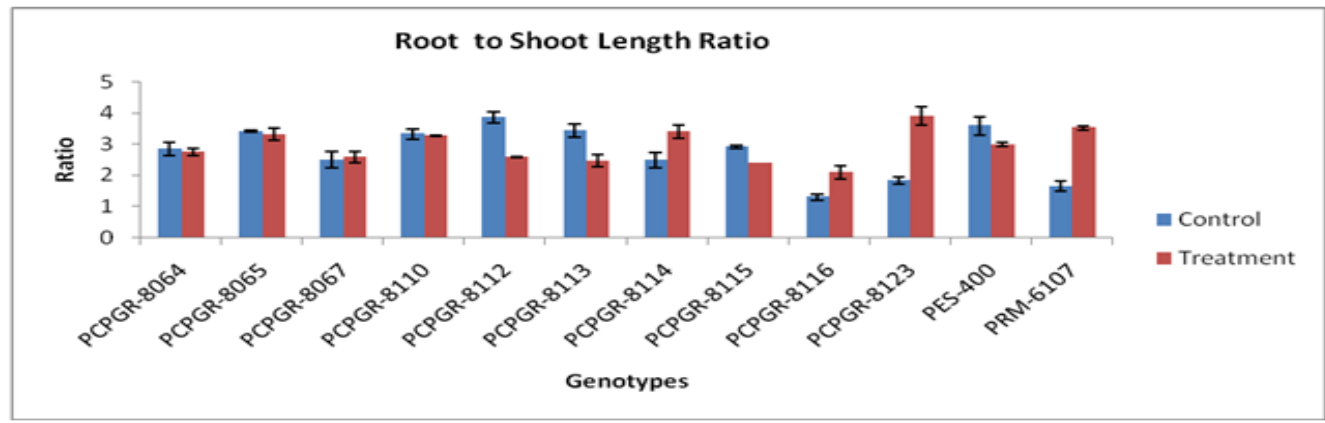

Fig.4 Effect of drought stress on number of tillers

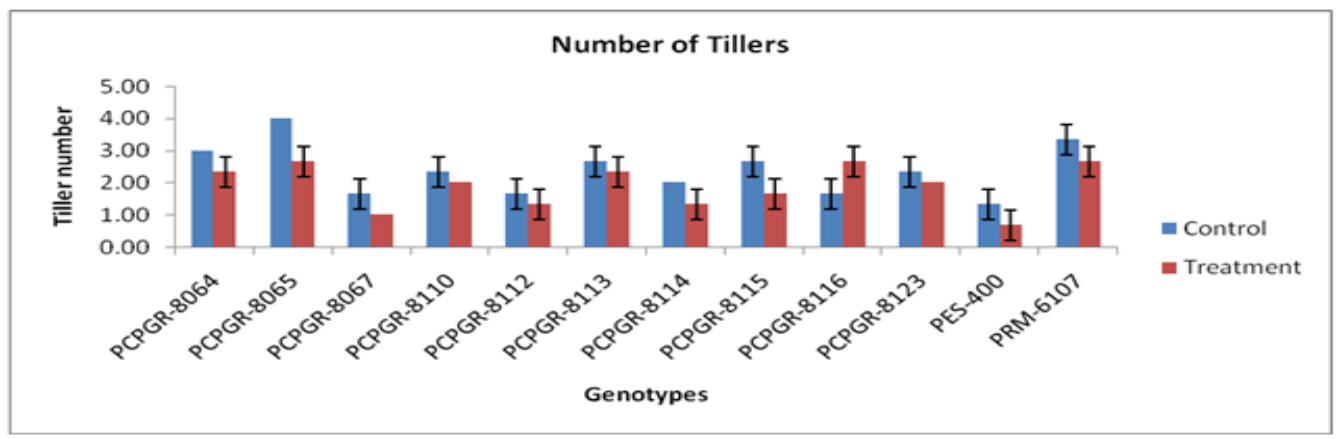

Fig.5 Effect of Drought stress on number of leaves

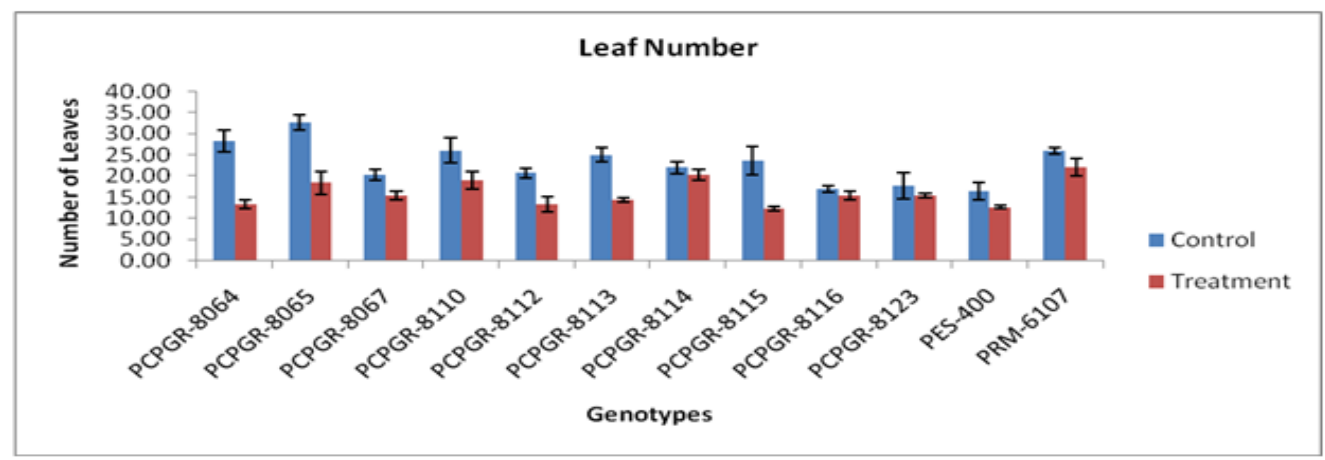


Fig.6 Effect of Drought stress on leaf area

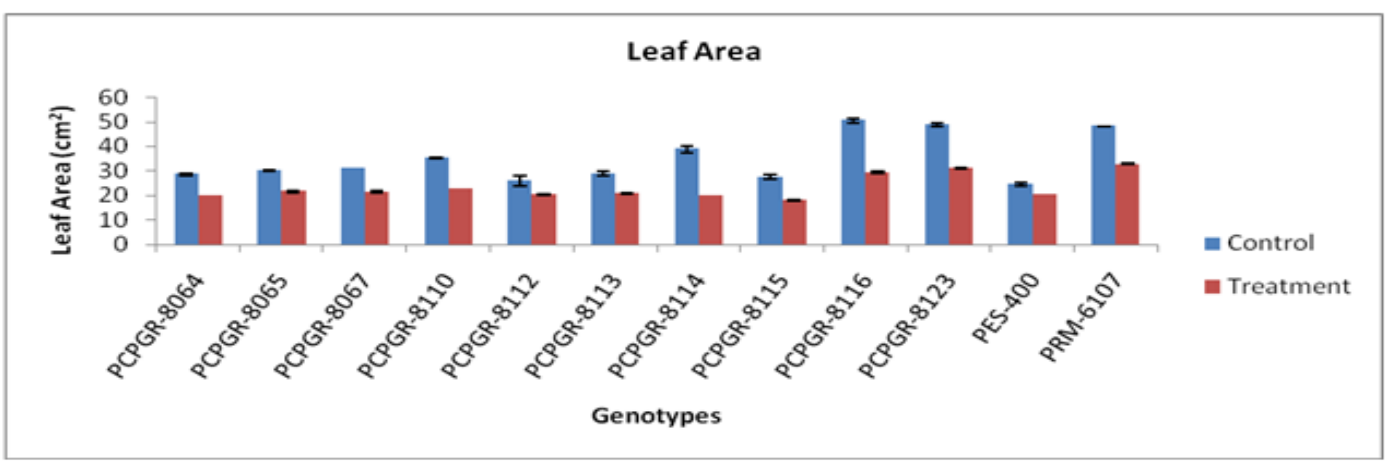

Fig.7 Effect of drought stress on relative water content

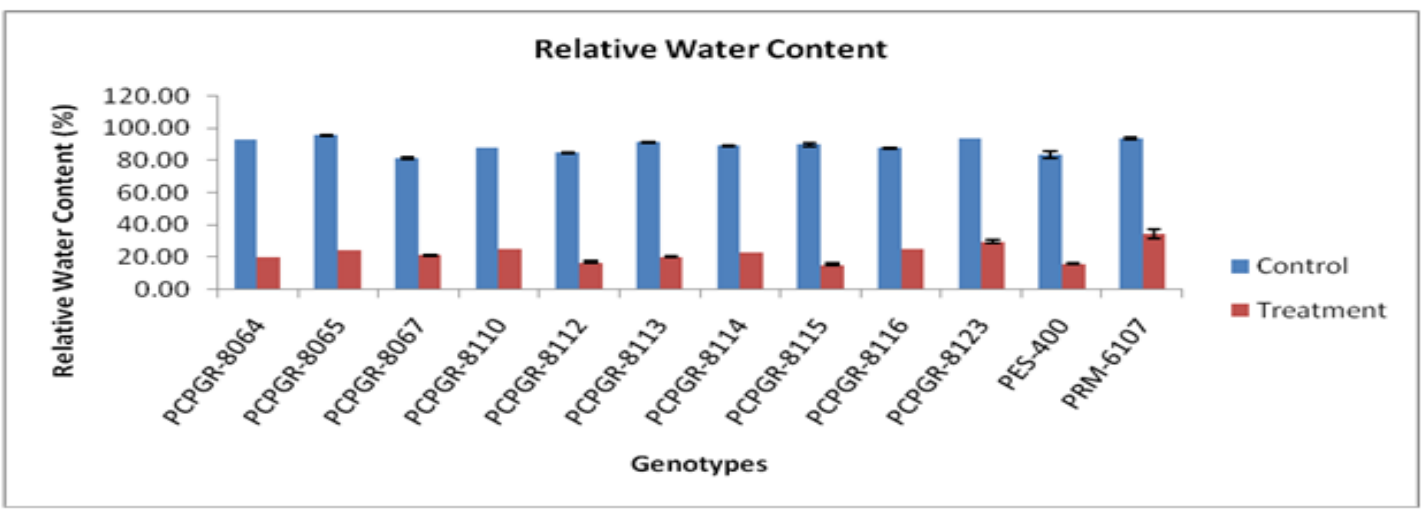

Fig.8 Effect of drought stress on total chlorophyll content

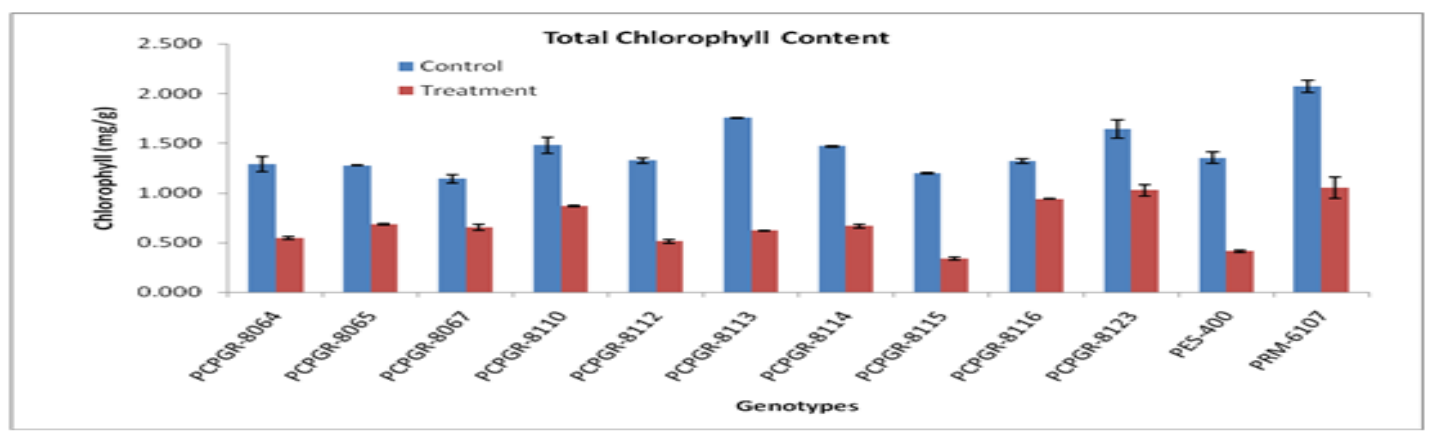


Fig.9 Effect of drought stress on total carotenoid content

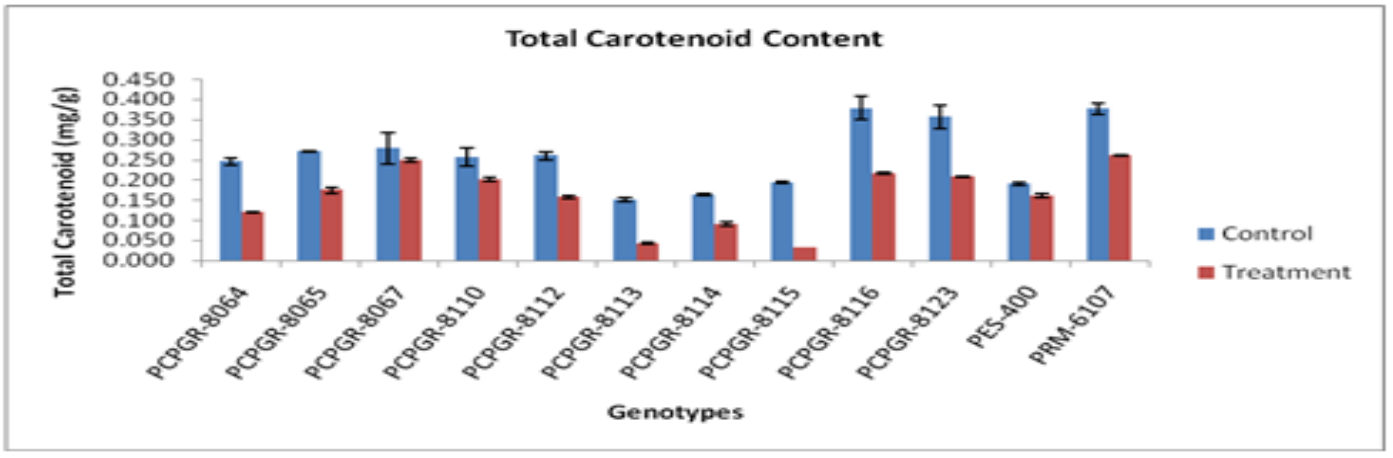

Fig.10 Effect of drought stress on proline content

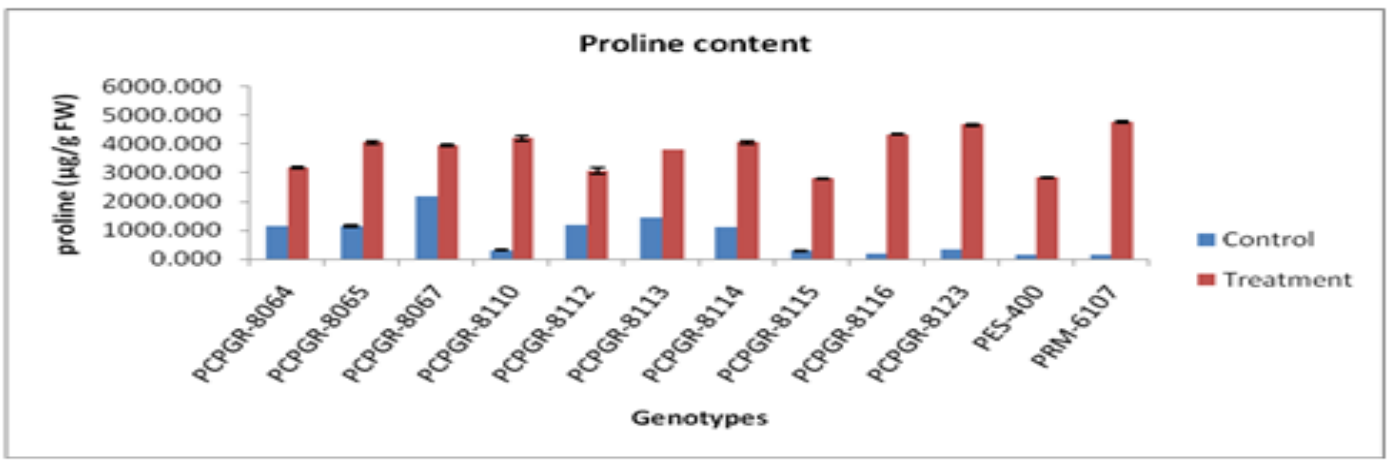

Fig.11 Effect of drought stress on malondialdehyde content

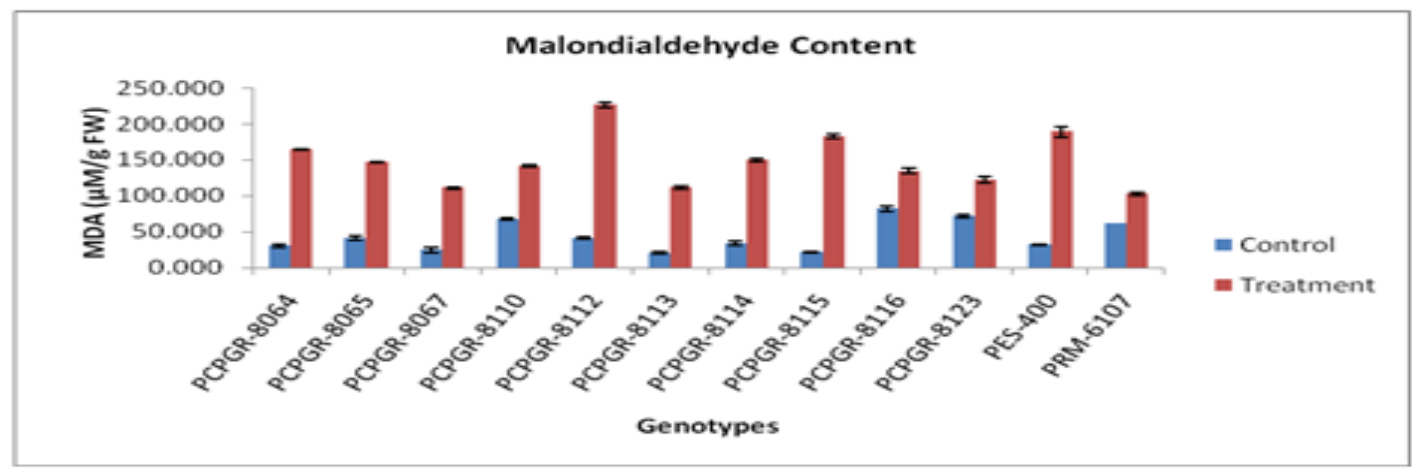


Fig.12 Percent increase in malondialdehyde content of drought treated plants over control plants

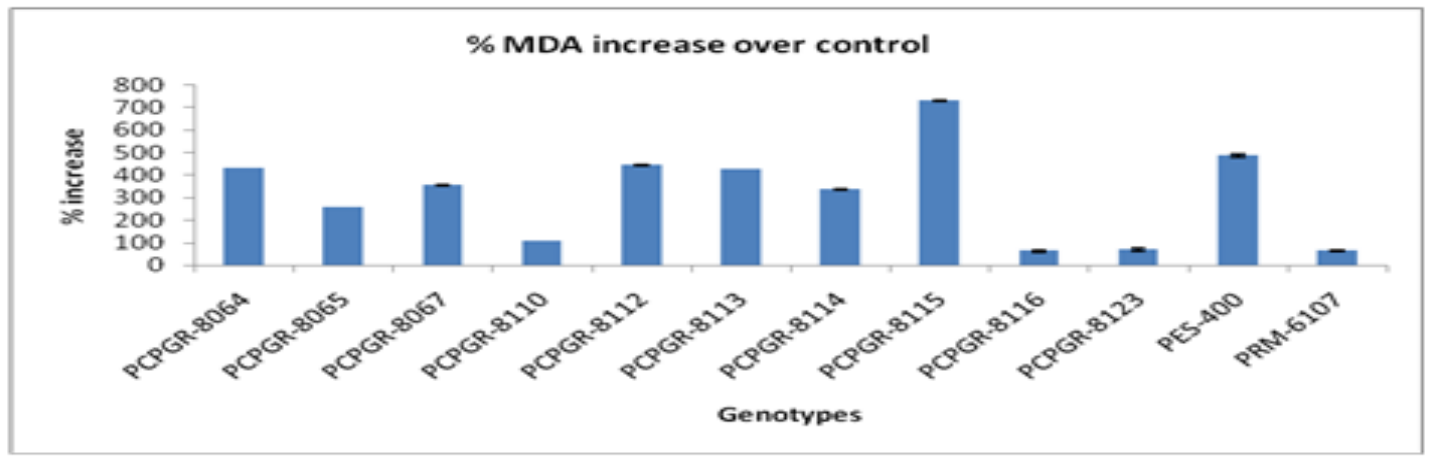

Fig.13 Agarose gel electrophoresis of isolated genomic DNA

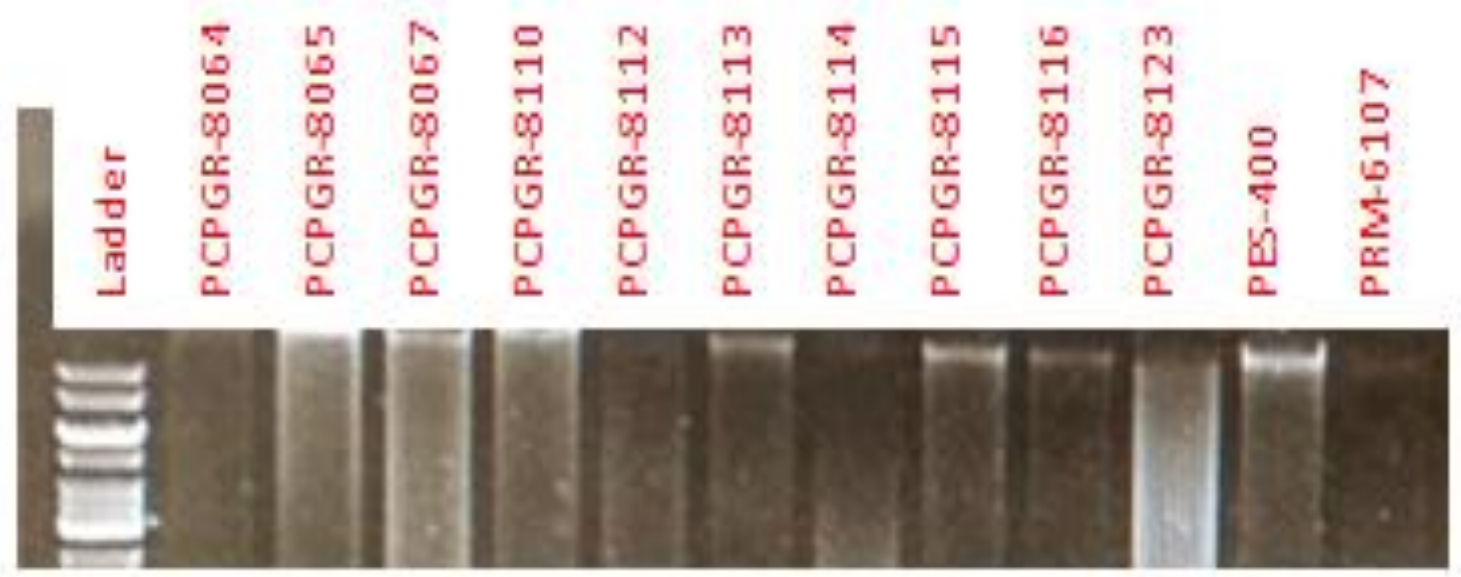

Fig.14 Agarose gel electrophoresis of PCR amplicons

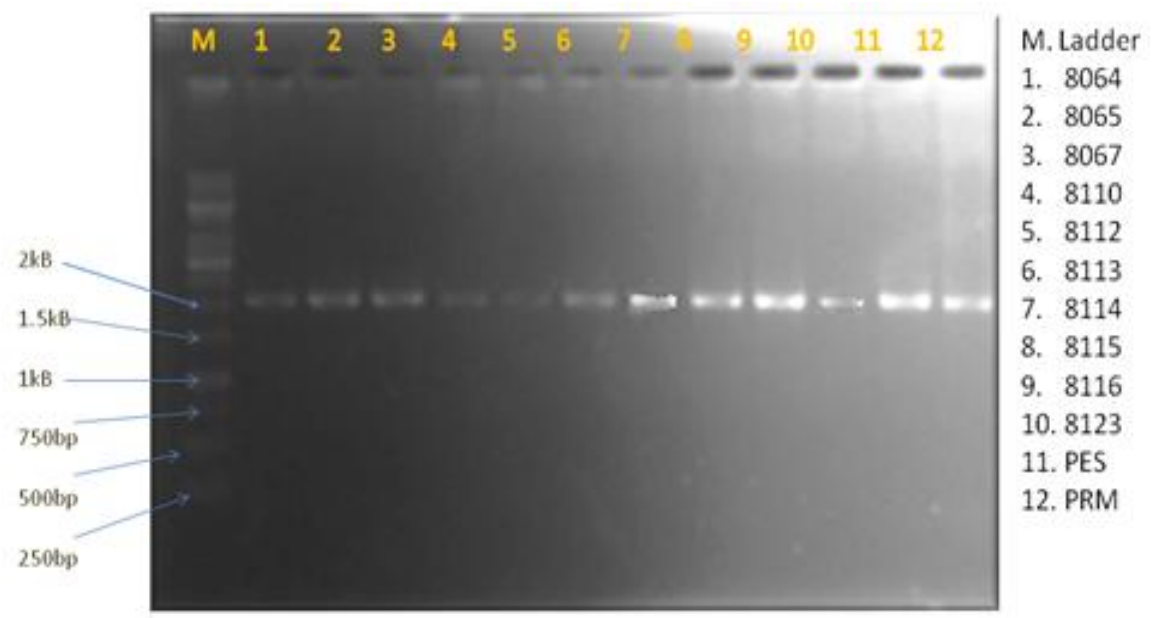


Fig.15 SNP observed at position 242

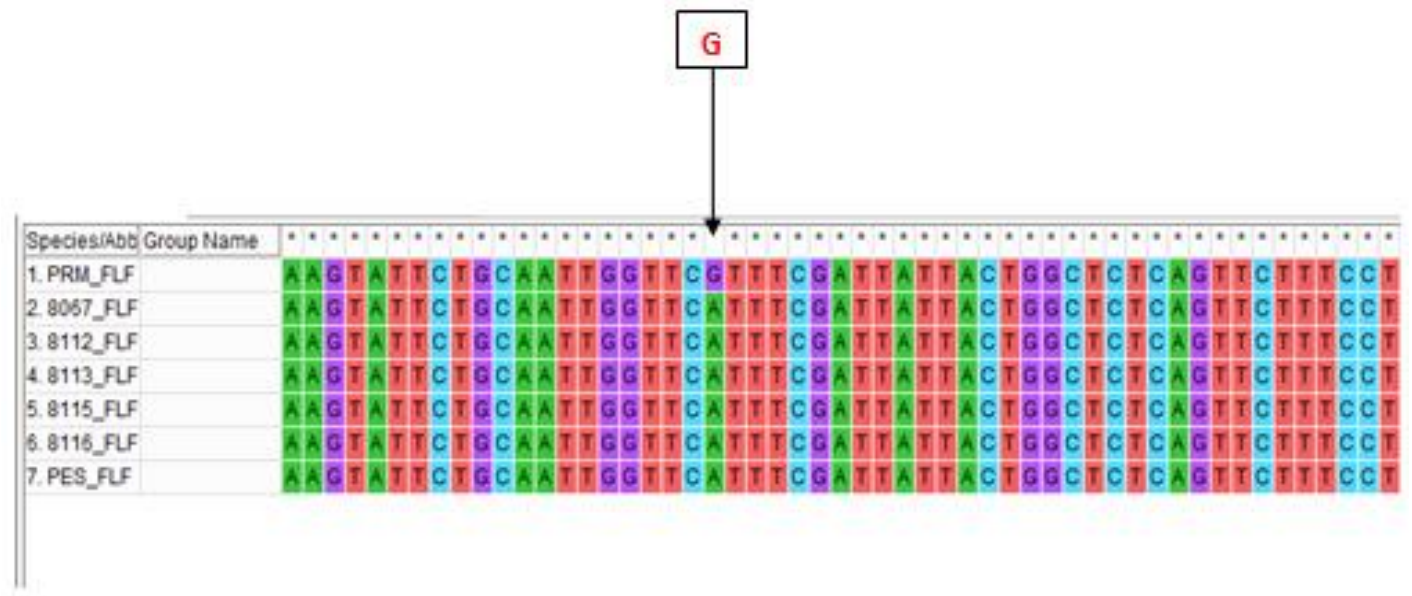

Fig.16 SNP observed at position 250

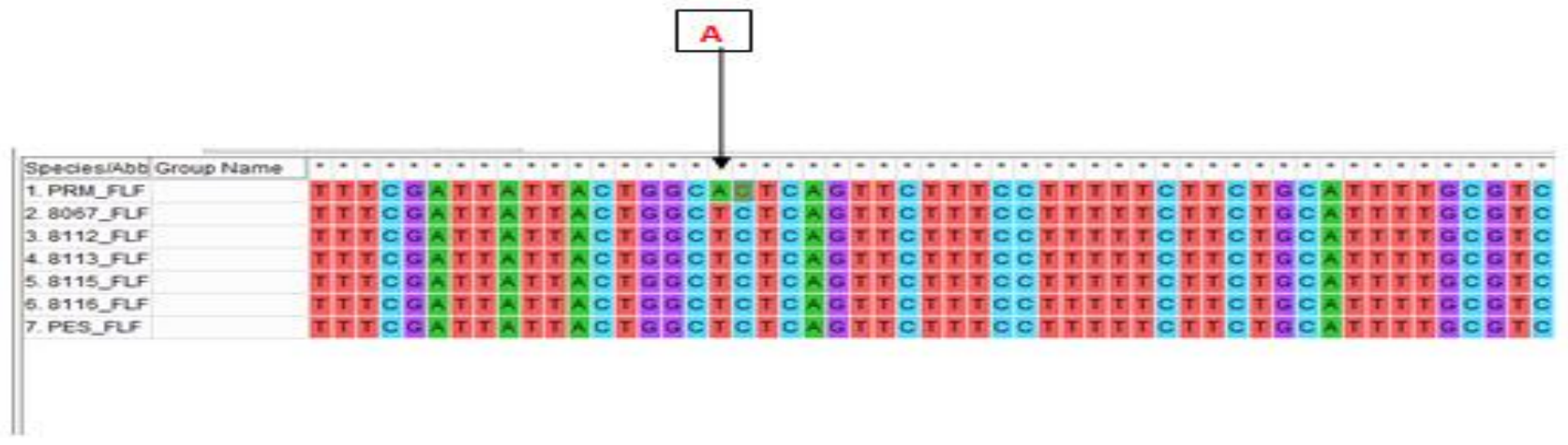

Fig.17 SNP observed at position 259

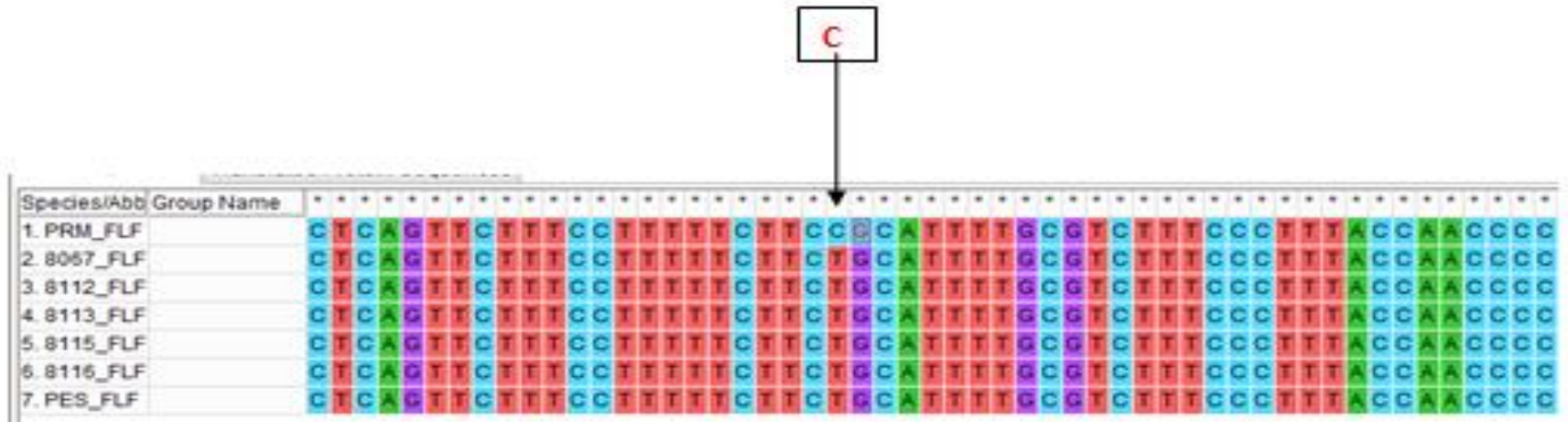


Fig.18 Insertion observed a position 311

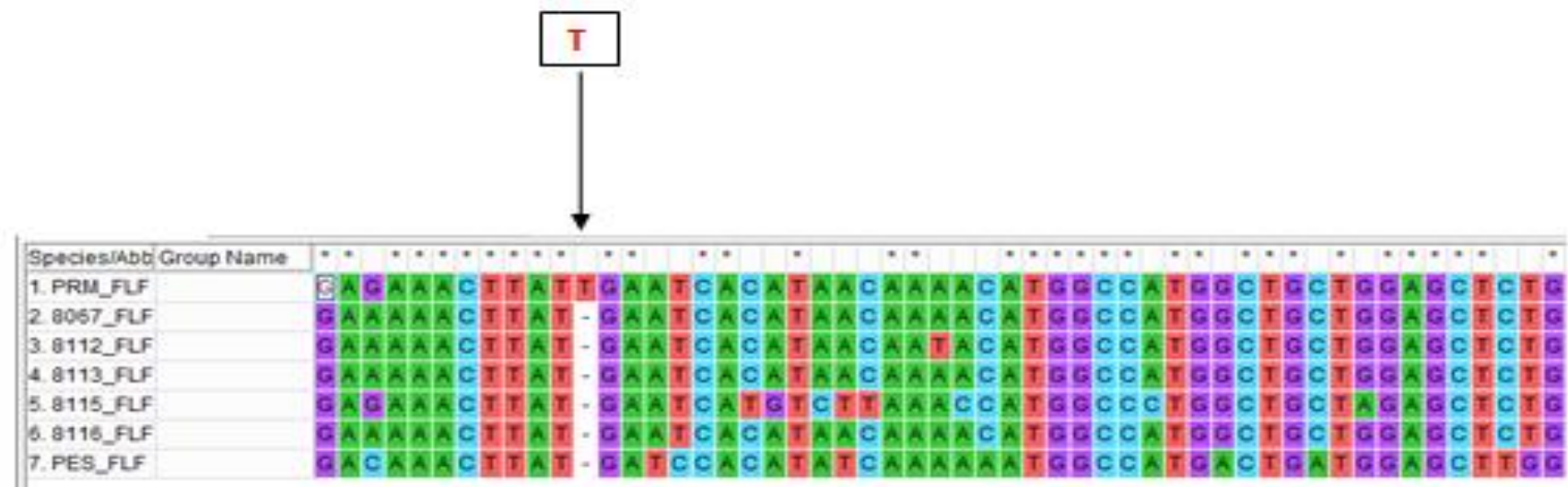

\section{Malondialdehyde content}

Detection of the degradation of poly unsaturated fatty acid products such as malondialdehyde is considered as an important criterion for determining the degree of stress effect in response to moisture deficit conditions in plants (Jain et al., 2001). In this study it was observed that different genotypes showed different responses with respect to the MDA content when exposed to drought conditions. The genotype PCPGR-8115 showed the highest MDA accumulation indicating that it has lower ability to maintain the cell membrane integrity while PRM-6107 showed the least MDA content indicating it has a better ability to counter oxidative stress generated as a result of drought conditions (Fig. 11, 12).

On the basis of the various morphophysiological and biochemical indices used to assess the drought tolerance of 12 genotypes of finger millet, the order of drought tolerance of these genotypes was found to be as follows:

PRM-6107 > PCPGR-8123 > PCPGR-8116 > PCPGR-8110 > PCPGR-8065 > PCPGR-8114 $>$ PCPGR-8067 > PCPGR-8113 > PCPGR-
$8064>$ PCPGR-8112 > PES-400 > PCPGR8115

Genomic DNA of all the genotypes was isolated. The DNA was purified and quantified spectrophotometrically. The quality was checked by running on $0.8 \%$ agarose gel (Fig. 13). PCR of all the isolated DNA was performed using gene specific primers and high fidelity DNA polymerase with proof reading ability. The PCR amplicons hence obtained were run on $1.2 \%$ agarose gel with $1 \mathrm{kB}$ marker. Fluorescent bands of around 2.1 $\mathrm{kB}$ were observed which were carefully eluted (Fig. 14).

The eluted PCR products were sequenced. Proper sequencing data could be obtained for 7 genotypes out of the 12 under study. This may be due to the fact that finger millet is an allotetraploid and hence has 2 alleles for a gene in its genome. This makes it possible for the primers to bind to both the alleles present in the genome if they have very similar sequences. Hence both the alleles get amplified resulting in faulty sequencing data. This problem can be addressed by cloning the PCR amplicons in suitable vectors and then sequencing them. 
Rest of the sequences hence obtained was subjected to In-silico analysis. Since PRM6107 was found to be the most drought tolerant genotype based on the results obtained from screening using the various morphophysiological and biochemical parameters, hence it can be safely hypothesized that it harbors the most potent Myb allele for drought tolerance. This allele can be considered as superior allele amongst the genotypes under study. Therefore all further study was performed focusing on theMyb allele sequences. Multiple sequence alignment for sequence analysis using the MEGA offline tool was performed using default as gap creation and gap extension penalty. The aligned sequence of PRM-6107 was manually analyzed for any InDel(Insertions and Deletions) and SNP (single nucleotide polymorphism). Multiple sequence alignment resulted in discovery of 3 SNPs and 1 insertion.

The 3 SNPs observed were at the position 242, 250 and 259 in the PRM-6107 Myb gene nucleotide sequence (Fig. 15-17). The insertion observed was at position 311 (Fig. 18).

\section{Multiple Sequence Alignment and Sequence Analysis}

Such nucleotide polymorphisms might be a possible reason for the differential response of the genotypes to drought stress. Singh et al., (2015), in their study on natural allelic diversity in OsDREB1F gene in rice observed a transversion in the coding region which was responsible for non-synonymous substitution and caused an amino acid change of aspartate into glutamate which is precursor of proline in plants. This they predicted was probably responsible for drought tolerance in wild rice accessions carrying the alleles. The most practical application of a nucleotide diversity study is to predict allelic selection on the drought tolerant genes and then to use Marker Assisted Selection (MAS) based on SNPs within the gene themselves to transfer the new alleles from wild or unadapted landraces into modern cultivars. Comparison of QTL and microarray data is difficult due to low number of sequence based markers in genetic map of crops such as wheat.

To overcome this problem SNP discovery is very important. On applied level, this very high density of SNPs has turned them into molecular markers of choice for fine mapping studies by most researchers (Mammadov et al., 2012). Apart from this, SNP study provides a framework for examining how population history, selection and breeding systems affect variation at genetic loci. This helps to delineate the mechanism that lead to evolutionary diversification of genomes (Nordborg and Innan 2002). SNPs provide the ultimate anchor to relate all forms of polymorphisms, including biochemical, metabolic, physiological and phenotypic performance (McNally et al., 2006).

The Insertions observed in this study too can have an important role, in molecular breeding programs particularly. The presence of insertions can be explained by the fact that some genotypes withstood a long term directional selection and changes that improved drought resistance were accumulated. Insertions thus obtained in this study can be used in marker anchored genetic map for identification of major QTLs governing candidate genes for drought tolerance. In order to validate the SNPs the PCR amplicon of PRM-6107 needs to be resequenced and checked for the presence of same nucleotides at the same observed positions

The new superior functional allele from PRM6107 was identified and isolated in this study. It can be concluded that after further 
validation studies, it may be transferred to susceptible but economically important varieties of food crops as single genes or gene cassettes.

\section{Acknowledgement}

The authors are thankful to the Department of Biotechnology, Govt of India for providing the financial support to Akash Sinha in the form of fellowship.

\section{References}

Barrs H. D. and Weatherley P. E., A reexamination of the relative turgidity technique for estimating water deficit in leaves, Aust. J. Biol. Sci., 15, 413428(1962)

Bates L. S., Waldren R. P. and Teare I. D., Rapid determination of free proline for water-stress studies, Plant and Soil,39, 205-207(1973)

Bhullar N. K., Zhang Z., Wicker T. and Keller B., Wheat gene bank accessions as a source of new alleles of the powdery mildew resistance gene Pm3: a large scale allele mining project, BMC Plant Biology, 10,88(2010)

Blum A., Drought resistance, water use efficiency, and yield potential are they compatible, discount, or mutual exclusive, Australian J. Research, 56, 1159-1168(2005)

Bouman B. A. M. and Toung T. P., Field water management to save water and increase its productivity in irrigated lowland rice, Agric. Water Manage.,49,11-30(2001)

Chakraborty U., Dutta S. and Chakraborty B.N., Response of tea plants to water stress, Biologia Plantarum,45(4),557562(2001)

Cortés A. J., This D., Chavarro C. et al., Nucleotide diversity patterns at the drought-related DREB2 encoding genes in wild and cultivated common bean (Phaseolus vulgaris L.), Theor. Appl. Genet., 125,069(2012)

Datta D. S. K., Malabuyoc J. A. and Aragon E.L., A field screening technique for evaluating rice germplasm for drought tolerance during the vegetative stage, Field Crops Research, 19(2),123134(1988)

Fang Y. and Xiong L., General mechanisms of drought response and their application in drought resistance improvement in plants, Cellular and molecular Life Sciences,72(4),673-689(2015)

Farooq M., Wahid A., Kobayashi N., Fujita D. and Basra S.M.A., Plant drought stress: effects, mechanisms and management, Agron. Sustain. Dev.,29,185-212(2009)

Heath R.L. and Packer L., Photoperoxidation in isolated chloroplast. I. Kinetics and stoichiometry of fatty acid peroxidation,

Arch.Biochem.Biophys.,125,189198(1968)

Hsiao T. C., O’Toole J. C., Yambao E.B. and Turner N.C., Influence of osmotic adjustment on leaf rolling and tissue death in rice (Oryza sativa L.), Plant. Physiol., 75, 338-341 (1984)

Inamullah Z. A., Swati A., and Siraj D., Evaluation of lines for drought tolerance in heat (Triticum aestivum L.), Sci.Khy. J.,12(2), 39-48(1999)

Jain M., Mathur G., Koul S., Sarin N.B., Ameliorative effects of proline on salt stress-induced lipid peroxidation in cell lines of groundnut (Arachis hypogaea L.), Plant Cell Rep.,20(5),463-468 (2001)

Jung C., Seo J. S., Han S. W., Koo Y. J., Kim, C. H. and Song S. I., Overexpression of AtMYB44 enhances stomatal closure to confer abiotic stress tolerance in transgenic Arabidopsis, PlantPhysiol., 146,623-635(2008) 
Kar R. K. and Sinhababu A., Comparative responses of three fuel wood yielding plants to PEG-induced water stress at seedling stage, Acta. Physiologiae Plantarum,25(4),403-409(2002)

Kashiwagi J., Krishnamurthy L., Crouch J. H. and Serraj R., Variability of root length density and its contributions to seed yield in chickpea (Cicer arietinum L.) under terminal drought stress, Field Crops Research,95(2),171-181(2006)

Kramer P. J., Plant and soil water relationships: a modern synthesis, McGraw-Hill Book Company, New York(1969)

Kumar S., Stecher G. and Tamura K., MEGA7: Molecular Evolutionary Genetic Analysis version 7.0. for bigger datasets, Molecular Biology and Evolution, 33,1870-1874(2016)

Ladizinsky G., Founder effect in crop-plant evolution, Econ. Bot.,39, 191199(1985)

Larbi A., Relative water content (RWC) and leaf senescence as screening tools for drought tolerance in wheat, In: Cantero-Martínez C. (ed.), Gabiña D. (ed.). Mediterranean rainfed agriculture: Strategies for sustainability. Zaragoza : CIHEAM, 193-196(2004)

Li Z. M., Zheng X. M. and Ge S., Genetic diversity and domestication history of African rice (Oryza glaberrima) as inferred from multiple gene sequences, Theor. Appl. Genet., 123,21-31(2011)

Lichtenthaler H. K. and Wellburn W. R., Determination of total carotenoids and chlorophylls a and b of leaf extracts in different solvents, Biochem. Soc. Trans., 11, 591-592(1983)

Mammadov J., Aggarwal R., Buyyarapu R., Kumpatla S., SNP markers and their impact on plant breeding, International journal of plant genomics 2012,(2012)
McNally K. L., Bruskiewich R., Mackill D., Buell C.R., Leach J.E. and Leung H., Sequencing multiple and diverse rice varieties. Connecting whole-genome variation with phenotypes, Plant Physiology, 141(1), 26-31(2006)

Murray M.G. and Thompson W.F., Rapid isolation of high molecular weight plant DNA, Nucleic acids research,8(19),4321-4326(1980)

Nordborg M. and Innan H., Molecular population genetics, Curr.Opin. Plant Biol., 5, 69-73(2002)

Pande H. and Singh J.S., Influence of clipping and water stress on growth performance and nutrient value of four range grasses, Indian Acad. Sci.,95,389-403(1985)

Potter J.R. and Jones J.W., Leaf area partitioning as an important factor in growth, Plant Physiology,59(1), 1014(1977)

Rajappa M. G., Jagannath M. K., Rajashekara B. G. and Mallana K. N., Leaf area determination in ragi or finger millet (Eleusinecoracana Gartn.),Mysore Journal of Agricultural Science, 6, 102-106(1972)

Rich S. M. and Watt M., Soil conditions and cereal root system architecture: review and considerations for linking Darwin and Weaver, J. Exp. Bot.,64, 11931208(2013)

Riechmann J. L., Heard J., Martin G., Reuber L., Jiang C., Keddie J., Adam L., Pineda O., Ratcliffe O. J., Samaha R. R., Creelman R., Pilgrim M., Broun P., Zhang J. Z., Ghandehari D., Sherman B. K. and $\mathrm{Yu}$ G., Arabidopsis transcription factors: genome-wide comparative analysis among eukaryotes, Science, 290, 2105-2110 (2000)

Salvi P., Arora S. and Lohani P., Expression of MYB Transcription Factor in Eleusinecoracana: towards making of 
drought tolerant plants, LAP Lambert Academic Publishing, (2012)

Shmat'ko I. G. and Shvedova O. E., Vodnyirezhimizasukhoustoichivost' pshenitsy (Water Regime and Wheat Drought Tolerance), Kiev: Naukova Dumka (1977)

Simmonds N. W., Evolution of Crop Plants. Longman, London,(1976)

Singh B. P., Jayaswal P. K. and Singh B., Natural allelic diversity in OsDREB1F gene in the Indian wild rice germplasm led to ascertain its association with drought tolerance, Plant Cell Rep., 34, 993(2015)

Srividya A., Vemireddy L. R., Ramanarao P.V., Sridhar S., Jayaprada M., Anuradha G. abd Siddiq E.A., Molecular mapping of QTLs for drought related traits at seedling stage under PEG induced stress conditions in rice, American Journal of Plant Sciences,2(02), 190(2011)

Sultan M. A. R. F., Hui L., Yang L.J. and Xian Z.H., Assessment of drought tolerance of some Triticum L. species through physiological indices, Czech $J$. Genet. Plant Breed.48(4), 17884(2012)

Tomar R. S. S., Tiwari S., Naik B. K., Chand S., Deshmukh R., Mallick N. and Tomar S. M. S., Molecular and morpho-agronomical characterization of root architecture at seedling and reproductive stages for drought tolerance in wheat, PloS one,11(6), e0156528(2016)

Turner N. C. and Begg J. E., Responses of pasture plants to water deficits, Plant relations in pastures. ed JR Wilson,5066(1978)

\section{How to cite this article:}

Akash Sinha, Megha Bhatt, Ankita Chauhan and Pushpa Lohani. 2021. Identification of Superior Allele for Drought Responsive Myb Gene in different Germplasms of Eleusine coracana through Morpho-Physiological, Biochemical and Sequence Diversity Analysis. Int.J.Curr.Microbiol.App.Sci. 10(04): 268-286. doi: https://doi.org/10.20546/ijcmas.2021.1004.028 